\title{
INDEX OF NAMES
}

Numbers in bold type refer to invited lectures, italic to contributed papers and ordinary print to the discussion and elsewhere.

Ables, H. D. 196

Abt, H. A. 58, 120, 219, 220

Adam, M. G. XVI

Adams, T. F. 8, 67, 99

Aizenman, M. L. 97

Alexander, J. B. 56

Allen, M. S. 7, 51

Aller, L. H. 198, 199

Andersen, J. 109, 203

Andrillat, Y. 155-156, 197

Arnett, W. D. 199

Auer, L. H. 49

Auman, J. R. 15

Ayres, T. R. 57

Azzopardi, M. 149

Baglin, A. 159

Barry, D. 58, 191, 192

Baschek, B. 3-15, 8, 14, 15, 45, 80, 116, 117, $119,125,136$

Becker, W. XVII, 184

Bell, R. A. 14, 17, 18, 23, 49-65, 85, 99, 109,

$152,167,183,203,208,212$

Bentolila, C. 223-259

Bidelman, W. 15, 19, 22, 44, 45, 90, 109, 116,

$119,120,123,124,125,127,151,202,205$, 213

Blackwell, D. E. 30

Blanc-Vaziaga, M. J. 59, 187, 188, 189, 210

Blanco, C. 163

Bode, G. 5

Boeshaar, G. 203

Böhm, K.-H. 8

Böhm-Vitense, F. 8, 9, 31

Bolton, C. T. 17-18

Bond, H. E. 57, 121, 123, 124, 125, 193

Boyle, R. J. 192

Branch, D. R. 49, 55, 56, 61, 64

Brosche, P. 168

Bues, I. 23-24

Burbidge, E. M. and G. R. 198

Burkhart, C. 157-159

Butler, D. 55, 184, 193, 194, 195, 202
Caloi, V. 97

Cannon, A. 220

Canterna, R. 184, 190, 195, 196

Carbon, D. F. 8, 31, 55. 59, 138

Canavaggia, R. 95-96

Cayrel, R. 41, 50, 59, 67, 71, 188, 209. 212

Cayrel de Strobel, G. 223-259. 50. 59, 71, 78. $96,125,146,151,173,174,188.202,211$. $212,223-259$

Chalonge, D. 143-146, 225

Christensen, C. 196

Claria, J. J. 73, 101-109

Clegg, R. I:. S. 49, 55, 61

Code, A. D. XVII

Conti, P. S. 67, 119, 159, 212

Couteau, P. 168

Cowley, C. R. 51, 118, 119

Crawford, D. L. 71-72, 82

Cromwell, R. 58, 191, 192

Cucchiaro, A. $177-180$

Dachs, I. 151

Da Silva, L. 23, 168, 215-218

Day, R. W. 57

Dearborn, D. S. 57, 63, 190

Delbouille, L. 60, 63

Delcroix, A. 165

Demarque, P. 97

Deutsch, A. 118, 212

Dickens, R. J. 50, 55, 61, 63

Divan, L. 143-146

Dubois, P. 149-152

Dufour, R. 199

Eggen, O. J. XVI, 23, 69, 115. 125, 167, 168 ,

$173,174.175,192,193,196,205.213$

Eriksson, W. C. 50, 59

Feast, M. N. 150, 151

Florsch, A. 149

Forrester, W. T. 197

Foy, R. 14, 50, 63, 64, 125, 167. 168. 173. 209-212 
Fry, M. A. 197

Frye, R. L. 147
Furenlid, K. 1. 64, 202

Irvine, N. 127

Irwin, A. W. 17-18

Janes, K. A. $102,105,186,187,189,190$

Garrison, R. F. 14, 17-18, 45, 82, 85, 116, Jaschek, C. 15, 45, 46, 113-125, 149-152, $127,153,180$

Gascoigne, S. C. B. 197

Gerbaldi, M. 68, 80, 180, 208

Gingerich, O. 31,138

Głebocki, R. 89

Golay, M. 122

Gordon, K. C. 135,136

Graham, J. A. 115,121

Greenstein, J. L. 23, 31, 42, 115, 117, 120, 205

Grenier, S. $215-218$

Grenon, M. 75-78, 173, 190

Griffin, R. 19-20, 54, 57, 58, 63, 64, 191, 192, 202, 213

Gros, M. 179

Gross, P. G. 101, 105, 106

Groth, E. J. 8, 152

Gustafsson, B. 8, 9, 31, 50, 52, 54, 56, 59, 61, $64,184,190,202,211$

Gyldenkerne, K. 42

Hack, M. 45, 63, 125, 127

Hagen, J. P. 127

Hall, D. N. R. 57

Hansen, L. 56, 173, 207

Harmer, D. L. 56, 190

Hartoog, M. R. 51

Hartwick, F. D. A. $60,195,196$

Hauck, B. 67-69, 80, 164, 223-259

Hauge, O. 51

Hearn, A. 14

Hearnshaw, J. 34, 49, 53, 55, 185, 190

Heasley, J. N. 97

Heck, A. $215-218$

Hejlesen, P. M. 39, 40, 42, 43, 97, 171

Helfer, H. L. $34,192,193$

Henry, R. C. 158

Hesser, J. E. 85,158

Holweger, H. 19-20, 50, 51

Honeycutt, R. K. 118, 119

Houk, N. 120, 122, 123, 127-128, 220

Houtgast, J. 57

Houziaux, L. 17, 127, 153, 155-156, 165

Hoyle, F. 203

Humphries, C. M. 161-165

Hunger, K. 7, 116

Hutching, J. B. 150

Hyland, A. R. 138, 139

Iriarte, B. 184

$$
\text { 177-180, 202, } 224
$$

Jaschek, M. 123, 149-152, 177--80, 224

Jenner, D. C. 199

Johnson, H. L. VII, 88, 90, 184, 224

Joly, M. 197

Jones, D. H. P. 194

Jung, J. 216

Kandel, R. 14, 15, 25, 46, 165

Keenan, P. C. XVI, 19, 45, 80, 121, 122, 123 , $127,147,203,219,220$

Keller, G. 121

Kellman, E. XVI

Kinman, T. D. 192,196

Kjaergaard, P. 56, 61, 173, 203, 207

Knuckles, C. F. 184

Kohlschütter, E. XVI

Kondo, Y. 34

Kraft, R. P. 55, 184, 193, 195, 196

Krishna Swamy, K. S. 6

Kron, G. E. 135, 136

Krupp, B. M. 57, 63

Kuchowicz, B. 119

Kurucz, R. L. 8, 31, 61, 138

Labs, D. 8

Lambert, D. L. 57, 63, 64

Larson, R. B. 196

Leckrone, D. S. 12

Leighton, R. B. 91, 224

Lindblad, B. 192

Luebke, W. R. 190

Luyten, W. 205

Lynden-Bell, D. 193, 196

Macau-Hercot, D. 177-180

MacConnell, D. J. 123, 124, 147

Mäckle, R. 19-20, 31, 174

Maeder, A. 46, 63, 97-99, 172, 208

Magnenat, P. 67, 68

Malaise, D. 178

Malaroda, S. 119

Martinet, L. 211

Maury, A. C. 220

Mayall, N. U. 192

Mayor, M. 207-208, 212, 216

McCarthy, M. F. 17, 63, 152, 156, 180, 203, 205

McClure, R. D. 60, 102, 107, 108, 186, 187 , $190,192,193,195,196,197$ 
McCuskey, S. W. 118, 119

Mendoza V., E. E. 79-80, 93, 127, 172

Meurs, E. J. A. 213

Mianes, P. 95-96

Mihalas, D. 3, 5, 7, 8, 11, 31, 49

Minnaert, M. H. 57

Mitchell, R. 184, 224

Moore, C. C. 57

Morel, M. 223-259

Morgan, W. W. XV-XIX, 45, 58, 63, 72, 83, $85,87,88,90,113,118,119,120,121,122$, $125,127,133,149,156,192,203,219-220$

Morton, D. C. 8, 11, 67, 99

Müller, E. A. 44, 51, 64

Münch, G. XVI, 117

Nandy, K. $161-165$

Nariai, K. 6, 118

Nassau, J. J. XVI, 121

Neugebauer, G. 91, 224

Newell, E. B. 101

Nicholson, W. 92

Nikolov, N. 95

Nissen, P. E. 8, 19, 49, 54, 63, 72, 81-83, 85, 199,211

Nordlund, A. 50, 59, 61

Noyes, R. W. 57

Ohlmacher, J. 61

Oinas, V. 50, 167, 185, 188, 190

Oke, J. B. 67

Osawa, K. 69, 118

Osborn, W. 19, 55, 60, 65, 69, 73, 101-109,

$127,195,211,213$

Osmer, P. S. 150, 152, 197

Osterbrock, D. XVII

Paczynski, B. J. 57

Pagel, B. 55, 56, 59, 184, 185, 192, 211

Pannekoek, A. 87

Parsons, S. 152

Pasinetti, L. E. 173-175

Patchett, B. 184, 185

Payne-Gaposchkin, C. 91-93, 203

Pecker, J. C. 3

Peimbert, M. 198, 199, 202

Penston, M. 185

Perrin, M.-N. 50, 167-172

Perry, C. L. 61, 71-72

Pesch, P. 22

Peterson, R. 188, 189, 202

Petford, A. D. 135, 140

Pettit, E. 92

Peytremann, E. 8, 9, 31, 49, 54, 61, 63, 177, 211
Philip, A. G. D. 101

Praderie, F. 159

Prather, M. J. 97

Preston, G. W. 120, 193

Przybylski, A. 56, 135-141, 151, 152, 197

Querci, F. 14, 25, 64

Racine, R. 97, 102

Raff, M. 190

Redman, F. 58, 184

Reimers, D. 8

Reza, de la, R. 14, 15, 80

Robinson, L. 193

Roman, N. G. XVI, 88, 90, 120

Rood, R. T. 60

Rosendahl, J. D. 150

Rousseau, J. 95-96

Rudkjфbing, M. 127, 159

Sandage, A. R. 69, 96, 192, 193, 196

Sanduleak, N. 73, 149, 203

Sargent, W. L. W. 115, 116, 117

Sauval, A. J. 21-22

Schatzman, E. 14, 85

Scheid, P. S. 216

Schild, R. 67

Schlesinger, B. M. 97

Schmidt, E. G. 95, 96, 122, 162

Scholz, M. 13

Schönberner, D. 8

Schwarzschild, M. and B. 192

Schweizer, F. 196

Searle, L. 115, 117, 119, 198

Seitter, W. 64, 115, 129-133

Sharpless, S. XVII

Shipman, H. L. 23

Slettebak, A. 115, 119, 121

Smethells, W. G. 205

Smith, H. E. 197, 198, 202

Smith, M. 159

Sneden, C. 55, 57, 193

Spinrad, H. 20, 45, 49, 57, 58, 80, 83, 96, 99, 141, 183-204, 210, 213

Spite, F. 34, 43, 215

Spite, M. 43, 216, 218

Steinlin, U. 43, 184, 202

Stephenson, C. B. 17, 73, 83, 203

Stock, J. 73

Stokes, N. R. 57

Stone, R. P. S. 197,198

Strittmatter, P. A. 23

Strohbach, P. 167

Strom, S. E. 8, 59, 138, 189 
Strömgren, B. XV-XVIII, 42, 61, 71, 81, 219 Sturch, C. 193

Swings, J. P. 177, 179

Talbot, R. J. 199

Tammann, G. A. 96

Taylor, B. J. 49, 57, 184, 189, 190, 191, 197, 210

Thompson, G. I. $161-165$

Thomsen, B. 83

Tinsley, B. 211

Titus, J. XVI, 119

Tomkin, J. 57, 64

Torres-Peimbert, S. 97, 199

Traving, G. 7, 13

Tsarevsky, G. S. 95

Tsuji, T. 21, 211

Unsöld, A. 3, 11, 143

Upgren, A. R. 125, 147

Upson, W. L. 57

Van den Bergh, S. 195, 196, 197

Van Paradijs, J. 213

Van 't Veer, Cl. 68, 157-159

Vauclair, S. 85, 159

Veth, C. 18
Walborn, N. 19, 44, 45, 83, 85, 116, 125, 133, $152,153,202$

Wallerstein, G. 34, 192

Wampler, E. J. 193

Warner, B. 34, 123, 198

Wegner, G. 23, 135, 140

Wehrse, R. 13, 25

Welch, G. 197

Westerlund, B. 96

Whitford, A. E. XVII

Whitney, C. A. 91

Wickramasinghe, N. C. 23

Wildey, R. L. 138

Williams, P. M. 56, 62, 64, 72, 87-90, 109, $125,127,147,173,175,183,184,189,190$, 202, 211, 213

Willstrop, R. V. 56

Wilson, O. C. $121,122,147$

Wing, R. F. 57

Wolf, B. 8, 151, 152

Woodrow, J. E. J. 15

Woollcy, R. 38

Wroblewski, H. 73

Yakimova, N. N. 95

Zinn, R. 55, 196 